Z. klin. Chem. u. klin. Biochem.

8. Jg., S. 299-301, Mai 1970

\title{
Quantitative Acetonbestimmung in der Atemluft
}

\author{
Von H. Göschke \\ Aus der Medizinischen Universitätsklinik, Bürgerspital Basel \\ (Vorsteber: Prof. Dr. F. Koller)
}

(Eingegangen am 16. Dezember 1969)

\begin{abstract}
Es wird eine einfache, kolorimetrische Methode zur quantitativen Acetonbestimmung in der Atemluft beschrieben. Gemischte Exspirationsluft wird durch ein Spirometer geleitet und in einem Douglassack gesammelt. Aus diesem wird die Atemluft durch zwei Gaswaschflaschen gesogen, welche 2,4-Dinitrophenylhydrazin-Lösung enthalten. Aceton wird quantitativ in Aceton-2,4-dinitrophenylhydrazon übergeführt, als solches in Tetrachlorkohlenstoff extrahiert und photometrisch bestimmt. Bei geringem apparativem Aufwand erreicht die beschriebene Methode Reproduzierbarkeit und Empfindlichkeit der gaschromatographischen Acctonbestimmung.

Bei 26 gesunden Personen wurde ein mittlerer Nüchternwert von 1,12 $\mu \mathrm{g}$ Aceton/l Exspirationsluft gefunden. Die Anwendung der Methode bei Ketosen wird anhand von Acetonbestimmungen gezeigt, welche bei adipösen Personen während vollständigen Fastens durchgeführt wurden.
\end{abstract}

\section{The quantitative determination of acetone in expired air}

A simple colorimetric method for the quantitative estimation of acetone in expired air is described. Mixed expired air is collected in a Douglas bag after passing through a spirometer. The content of the sampling bag is drawn by a pump through an acetone trap, which consists of two bubblers containing 2,4-dinitrophenylhydrazine solution. The acetone-2,4-dinitrophenylhydrazone formed is extracted into carbon tetrachloride, and the optical density is determined. While applying simple and inexpensive equipment, reproducibility and sensitivity of the present method were found to be comparable to those reported for gas chromatographic acetone estimation.

A mean value of $1.12 \mu \mathrm{g}$ Acetone/l of expired air was found in 26 normal subjects fasted overnight. To illustrate the application of the procedure to ketosis, acetone values obtained during total starvation of obese subjects are reported.

Von Greenberg wurde eine Methode zur kolorimetrischen Bestimmung des Acetongehaltes in der Luft mitgeteilt, welche für fabrikhygienische Untersuchungen bestimmt war (1). Diese Methode zeichnet sich durch eine hohe absolute Empfindlichkeit aus, indem Mengen in der Größenordnung von $0,1-0,2 \mu \mathrm{g}$ noch meßbar sind. In ein evakuiertes Gefäß, welches 2,4-Dinitrophenylhydrazin-Lösung enthält, wird die zu untersuchende Luft eingesogen. Aceton reagiert mit 2,4Dinitrophenylhydrazin unter Bildung des entsprechenden Hydrazons, welches in Tetrachlorkohlenstoff extrahiert und photometrisch bestimmt wird.

Die Methode von GreENBERg, welche sich für Untersuchungen an Patienten wenig eignet, wurde von GlaUBITr für klinische Zwecke modifiziert (2). Hierbei wird gemischte Exspirationsluft gesammelt und ihr Volumen gemesser, indem ein Atembeutel mit bekanntem Fassungsvermögen vom Patienten vollständig mit Atemluft gefüllt wird. Der Inhalt des Atembeutels wird anschließend durch eine Glastöhre in ein Reagenzglas mit 2,4-Dinitrophenylhydrazin-Lösung ausgepreßt. $\mathrm{Zu}$ letzt wird wie bei GREeNBERG das gebildete Hydrazon extrahiert und photometrisch bestimmt.

Die Methode von GlauBrTt war als klinische orientierende Untersuchung gedacht und leistet als solche ausgezeichnete Dienste. Für metabolische Untersuchungen ist die Methode jedoch weniger geeignet, weil damit das Aceton nach unseren Erfahrungen nicht quantitativ erfaßt wird. Die von Glaubirt gefundenen Nüchternwerte (bis zu 0,2 $\mu \mathrm{g}$ Aceton/l gemischter Exspirationsluft) sind denn auch wesentlich niedriger als die von anderen Autoren angegebenen mittleren Nüchternwerte von $1,1 \mu \mathrm{g} / /$ (3) und $1,04 \mu \mathrm{g} / /$ Alveolarluft (6) ${ }^{1}$ ).

Ausgehend von den Angaben von Greenberg und von GlaubitT wurde eine einfache Methode zur quantitativen Bestimmung des Acetons in der Atemluft ausgearbeitet. Sie entspricht hinsichtlich Reproduzierbarkeit und Empfindlichkeit der gaschromatographischen $\mathrm{Me}-$ thode, arbeitet aber mit geringerem apparativem Aufwand. Weil die Gewinnung von Alveolarluft klinisch oft nicht möglich ist (2) und weil mit der Alveolarluft nicht die gesamte Acetonabgabe erfaßt würde, erfolgt die Bestimmung in der gemischten Exspirationsluft.

\section{Methodik \\ Material}

Zum Sammeln der Atemluft wurden Douglassäcke von etwa $75 l$ Inhalt verwendet. Die Messung des Atemluft-Volumens erfolgte mit WRIGHTschen Spirometern, welche regelmäßig geeicht wurden. Der erwähnte Spirometertyp ist in den meisten Kliniken verfügbar und nimmt dank seiner geringen Größe kaum Aceton auf. Die verwendeten $G$ aswaschflaschen hatten eine Länge von $30 \mathrm{~cm}$, eine Weite von $3,5 \mathrm{~cm}$ und cnthielten Glasfritten mit einer Porengröße von $90-150 \mu \mathrm{m}$.

\section{Reagenzien}

0,2proz. Lösung von 2,4-Dinitrophenylhydrazin (p. a.) in $5 \mathrm{~N}$ Schwefelsäure (p. a.). Diese Lösung wird zur Erniedrigung des Leerwertes dreimal mit Tetrachlorkohlenstoff ausgeschüttelt. Tetrachlorkohlenstoff (p. a.).

$0,5 \mathrm{~N}$ Natronlauge (p. a.).

Aceton (p. a.).

1) Zwischen der Acetonkonzentration in der Alveolarluft und der gemischten Exspirationsluft fand sich nach Graubirt kein Unterschied (2). 


\section{Durclfillbrung}

Die zu sammelnde Exspirationsluft wird durch ein Ventil und durch das anschließende, röhrenförmige Spirometer in den Douglassack ausgeatmet. Sobald das benötigte Luftvolumen ${ }^{2}$ ) gesammelt ist, wird der Inhalt des Douglassackes mit einer Pumpe durch zwei aufeinanderfolgende Gaswaschflaschen gesogen. Diese enthalten je $75 \mathrm{ml}$ Farbreagenz und stehen in einem Eisbad. Aceton wird beim Passieren der Gaswaschflaschen quantitativ in Aceton-2,4-dinitrophenylhydrazon übergeführt, sofern die Durchflußrate der Atemluft nicht mehr als 2,5 //Min. beträgt.

Anschließend wird der Inhalt beider Gaswaschflaschen zusammengegossen. Ein aliquoter Teil dieses Gemisches wird mit einem Drittel seines Volumens Tetrachlorkohlenstoff während $20 \mathrm{Min}$. ausgeschüttelt, wodurch das Hydrazon des Acetons extrahiert wird. Nach dem Absetzen des Tetrachlorkohlenstoffs saugt man die wäßr. Schicht ab. Hierauf wird ein aliquoter Teil der Tetrachlorkohlenstoff-Fraktion mit dem $11 / 2$ fachen Volumen 0,5 proz. Natronlauge während $3 \mathrm{Min}$. ausgeschüttelt. Dadurch wird die Interferenz von Formaldehyd ganz und jene von Acetaldehyd zum größten Teil eliminiert, und die Farbintensität von Aceton-2,4-dinitrophenylhydrazon wird verstärkt (1). Nach dem Schütteln läßt man die Proben etwa 5 Min. stehen und saugt dann die wäßr. Schicht vollständig ab.

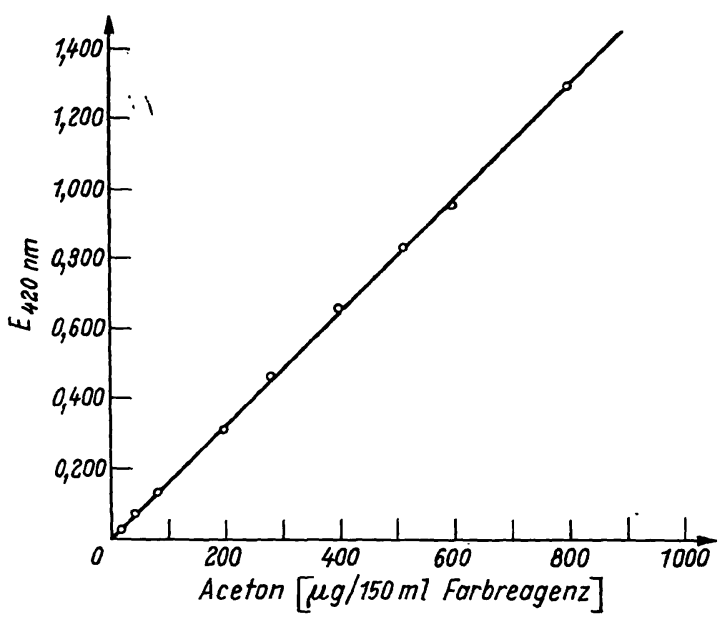

Abb. 1

Wiederfindungsrate von Aceton. Eichkurve: Angaben für $150 \mathrm{ml}$ Farbreagenz aus 2 Waschflaschen. $100 \mu \mathrm{g}$ Aceton in $150 \mathrm{ml}$ Farbreagenz entsprechen $2 \mu \mathrm{g}$ Aceton in $1 \mathrm{ml}$ Tetrachlorkohlenstoffextrakt. Schichtdicke $1 \mathrm{~cm}$

Die photometrische Bestimmung des Aceton-2,4-dinitrophenylhydrazons erfolgt für Werte im Normalbereich bei $375 \mathrm{~nm}$, für erhöhte Werte bei $420 \mathrm{~nm}$. Die Ablesung erfolgt gegen Tetrachlorkohlenstoff. Bei $420 \mathrm{~nm}$ und $1,0 \mathrm{~cm}$ Schichtdicke bewirkt $1 \mu \mathrm{g}$ Aceton pro $\mathrm{m} l \mathrm{MeBlösung}$ eine Extinktion von rund 0,080 (vgl. Abb. 1); bei $375 \mathrm{~nm}$ ist dieser Wert ungefähr doppelt so groß. Die Extinktion des Leerwertes entspricht im Mittel 0,2 $\mu \mathrm{g}$ Aceton pro $\mathrm{ml}$ Meßlösung. Proben, welche wegen zu hoher Hydrazonkonzentration nicht ablesbar sind, werden mit Tetrachlorkohlenstoff verdünnt.

Die gesamte, in beiden Gaswaschflaschen absorbierte Acetonmenge wird errechnet und diese durch das gemessene Luftvolumen geteilt, woraus die Konzentration pro $l$ Atemluft resultiert. Analog errechnet sich die Acetonabgabe pro Minute.

\section{Resultate}

\section{Wieiderfindungsrate}

Zur Ermittlung des prozentualen Acetonverlustes wurde Luft mit bekanntem Acetongehalt zubereitet und in den

2) Bei Acetonkonzentrationen im unteren Normbereich werden etwa 40 l Atẹmluft benötigt.
Douglassack eingebracht. Die mit steigenden Acetonmengen erhaltene Eichkurve (Abb. 1) zeigt einen geradlinigen Verlauf. Beim Vergleich mit einer Standardkurve, welche durch direkte Zugabe von Standardlösungen zum Farbreagenz erstellt wurde, zeigte sich jedoch ein Acetoniverlust von durchschnittlich 4,7 $(3,4-6,7) \%$. Als Ursache dieses Verlustes fand sich die Kondensation von Aceton im Atembeutel. Auch vom Acetongehalt der Atemluft blieben durchschnittlich $5,2 \%$ im Douglassack zurück. Bei allen Bestimmungen wurden zur Korrektur dieses Verlustes 5\% zum gefundenen Wert hinzugezählt.

\section{Normaliverte}

Bei 26 nüchternen Normalpersonen fand sich ein Wert $(\overline{\mathbf{x}} \pm \mathrm{s})$ von $1,12 \pm 0,63 \mu \mathrm{g}$ Aceton/l gemischter Exspirationsluft. Die Einzelwerte lagen zwischen 0,35 und $2,61 \mu \mathrm{g} / l$.

\section{Fastenketose}

Bei adipösen Personen wurde der Acetongehalt in der Exspirationsluft während vollständigem Fasten bestimmt. Der Verlauf der Acetonabgabe währeñ einer vierwöchigen Fastenperiode wird anhand von drei Beispielen in Abbildung 2 dargestellt.

\section{Diskussion}

Mit der beschriebenen Methode lassen sich in der Atemluft sowohl die Acetonkonzentration als auch die pro Minute abgegebene Acetonmenge bestimmen.

Die verwendete Gesamtmenge von 2,4-Dinitrophenylhydrazin reicht aus, um das Aceton der Exspirationsluft auch bei stark erhöhter Konzentration quantitativ zu erfassen. Die niedrigste meßbare Konzentration beträgt $0,2-0,3 \mu \mathrm{g}$ Aceton/l, was annähernd der für die Gaschromatographie angegebenen Nachweisgrenze von $0,1-0,3 \mu \mathrm{g} / \mathrm{l}$ entspricht $(3,4)$. Auch die mittlere Variation der Doppelbestimmungen, welche in einschlägigen Arbeiten als $\mathrm{Ma}$ für die Präzision angegeben wird, liegt mit 5,6\% in dem für gaschromatographische Methoden mitgeteilten Bereich $(3,4)$.

Als interferierende Substanzen kommen in der Atemluft Acetaldehyd (1), Formaldehyd (1) und Paraldehyd (2) in Frage. Wie bereits erwähnt, werden durch Alkalisierung Formaldehyd-2,4-dinitrophenylhydrazon vollständig und das entsprechende Derivat des Acetaldehỳds teilweise aus dem Tetrachlorkohlenstoff extrahiert. In normalen Konzentrationen verursachte Acetaldehyd in der vorliegenden Methode keine Störung; erst eine Konzentration von $5 \mu \mathrm{g} / \mathrm{l}$, wie sie nach. Alkoholgenuß vorkommt (5), führte zu einer meßbaren Extinktionszunahme, welche $0,3 \mu \mathrm{g}$ Aceton/l entspricht.

Der von uns bei 26 nüchternen Personen gefundene Mittelwert von 1,12 $\mu \mathrm{g}$ Aceton/l Exspirationsluft liegt im Bereich der gaschromatographisch bestimmten Normalwerte: Rooth fand bei 67 Personen im post- 


\section{Analytica70}

\section{Internationale Fachausstellung}

für Biochemische Analyse

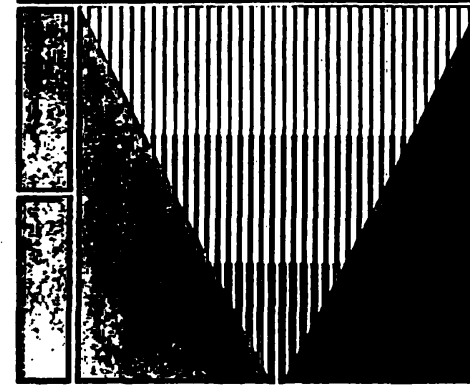

\section{mit Tagung:}

Biochemische Anallyitik

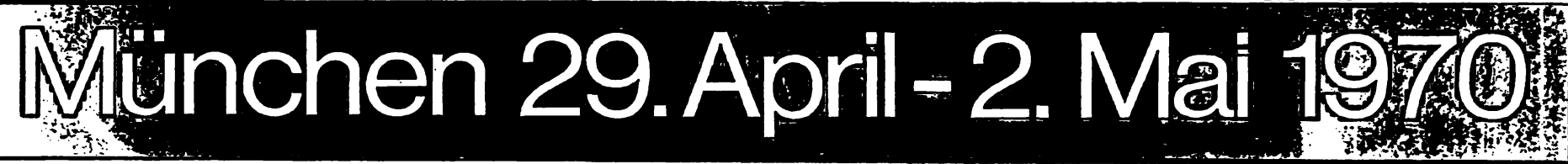

Auskunft: Münchener Messe- und Ausstellungsgesellschaft mbH · 8000 München 12,Theresienhöhe 13 ·Postfach 200 · Telefon (0811) 76711·Telex:05-212086 ·TA:AMEG

\section{labor einrichtegen}

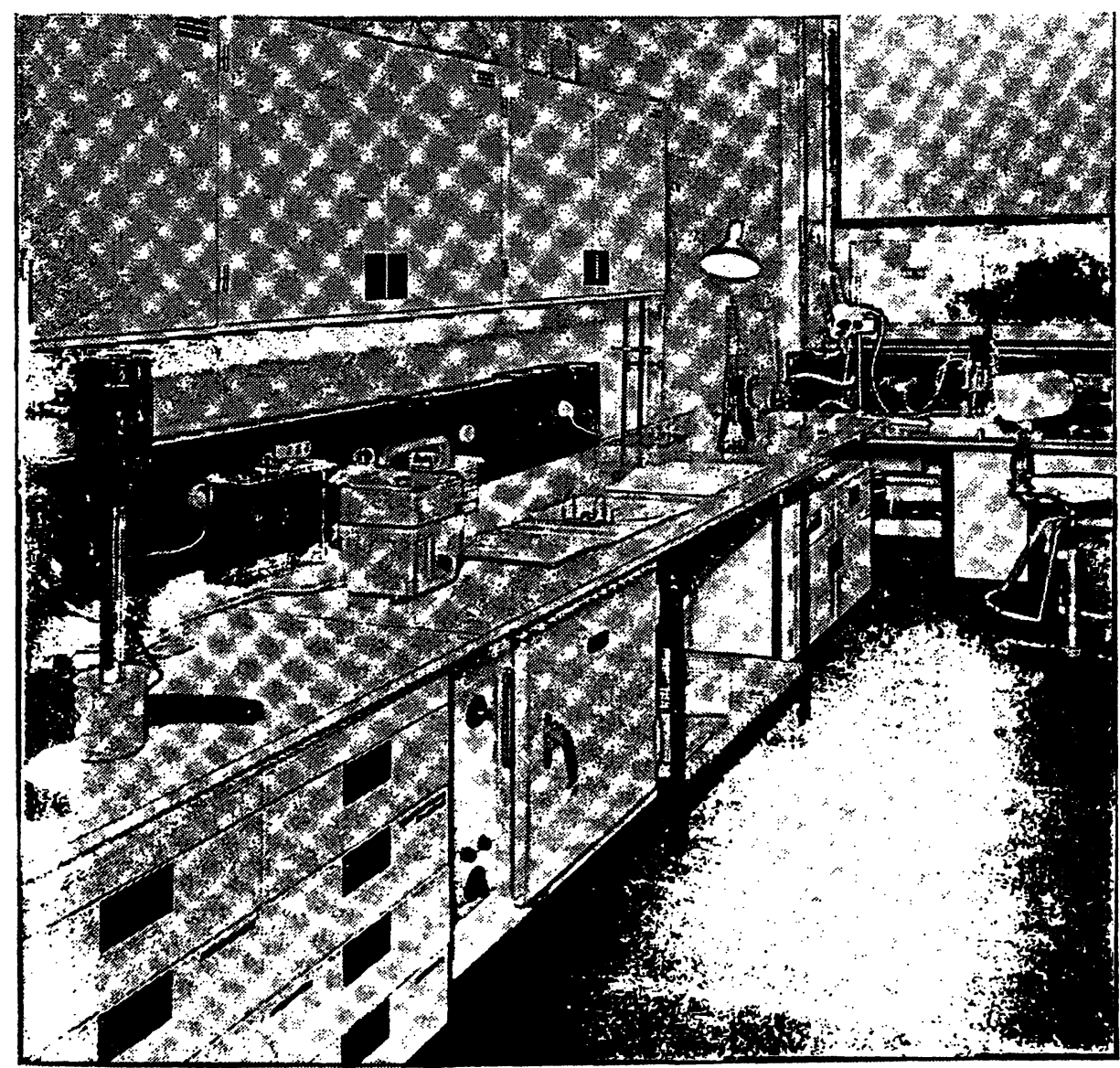

Die Errichtung eines modernen

Laboratoriums erfordert sorgfältige

Planung, die schon im Detail

frühzeitig einsetzen muß. Die Fachrichtung, die Aufgabenstellung und die räumliche Gruppierung der notwendigen Apparaturen bestimmen die Größe des Labors und den Umfang der Einrichtung.

Planen Sie die Neueinrichtung, Ergänzung oder Umstellung eines Labors - gleich welcher Fachrichtung und Aufgabenstellung wenden Sie sich bitte an uns.

Ein erfahrener Mitarbeiterstab steht Ihnen unverbindlich zur Beratung und Planung zur Verfügung.

Fordern Sie Prospekte an.

\section{WWh/h/, Burger Eisenwerke Akno Aktiengesellschaft Abt.Laboreinrichtungen 6348 Herborn}


aus Kunststoff

Einweg-Laborartikel

Reagenzröhrchenständer

Feuchtkammer

zur Blutgruppenbestimmung

Zentrifugierhilfe als Trennmittel

zur Gewinnung von

erythrocytenfreiem Serum

und Plasma

Dosierapparat zum Einfüllen

des Trennmittels in

Zentrifugenröhrchen

\title{
WALTER SARSTEDT KUNSTSTOFF.SPRITZGUSSWERK 5221 R O M MELS D ORF BEZ. KOLLN TEL. 02293/7133 und 385 - TELEX 884916
}

\section{Untallohirurgisohe Operationer}

\author{
Indikation Technik Fehler
}

\author{
von Dr. med. Erich Jonasch
}

2., erweiterte und verbesserte Auflage mit einem Geleitwort von Prof. Dr. med. Lorenz Böhler Quart. XII, 143 Seiten. Mit 121 Abbildungen in 315 Einzeldarstellungen. 1970. Plastikeinband DM 48, -

Die zweite, erweiterte Auflage bringt die Darstellung never, bewährter Operationsverfahren bei Verletzungen des Schädels, der Wirbelsäule und der Körperhöhlen.

Die Technik der einzelnen Operationen wird ausführlich beschrieben und durch zahlreiche schematische Zeichnungen und Röntgenbilder erläutert, so daß für den Operateur eine schnelle und ausführliche Orientierung möglich ist.

Bei jeder Operation ist auch die Indikation zur Operation angeführt.

Neben den typischen Eingriffen werden bei jedem Abschnitt häufig beobachtete Fehler, seien sie technischer oder indikationsmäßiger Art, besprochen und ihre Ursachen aufgezeigt.

Das kurz und prägnant gefaßte Buch bringt somit einen höchst aktuellen und interessanten Beitrag zur Frage der operativen Knochenbruchbehandlung und eine Übersicht über die notwendigen typischen unfallchirurgischen Operationen, die im Unfallkrankenhaus Wien $X X$ bei Professor Böhler durchgeführt werden.

Die Einstellung der Böhler-Schule in der Knochenbruchbehandlung ist bekanntlich konservativ, und es wird nur operiert, wenn eine konservative Behandlung allein nicht zum Ziel führt.

Wie großeReihen von Nachuntersuchungen zeigten, haben sich diese unfallchirurgischen Operationen bewährt. 


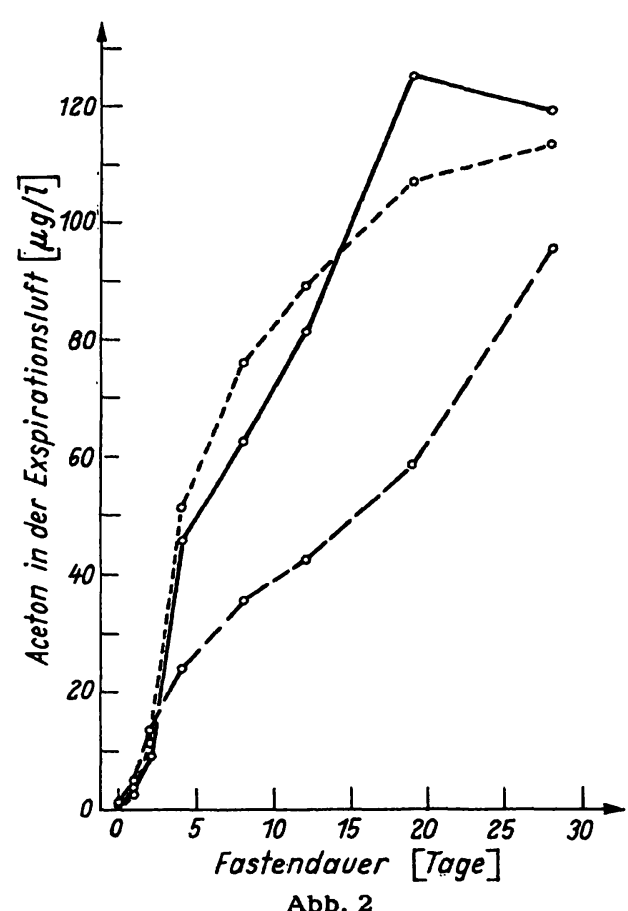

Abb. 2

Acetonkonzentration in der gemischten Exspirationsluft von drel adipösen Frauen während einer vierwöchigen Fastenperiode absorptiven Zustand einen Mittelwert $( \pm s)$ von $1,1+$ $0,88 \mu \mathrm{g}$ Aceton/l Alveolarluft (4). STEWART ermittelte bei 40 nüchternen Normalpersonen ebenfalls einen Wert von $1,1+0,50 \mu \mathrm{g}$ Aceton/l Alveolarluft (3). Unter gleichen Bedingungen erhielten TAssopoulos und Mitarbeiter bei 23 Personen einen Mittelwert von 1,04 \pm $0,29 \mu \mathrm{g}$ Aceton/l (6).

Úber den Acetongehalt der Atemluft während längeren Fastens finden sich in der Literatur nur wenige Angaben. Roort fand bei einer adipösen Patientin nach 14 tägigem Fasten eine Konzentration von $85 \mu \mathrm{g}$ Aceton/l und nach 21 tägigem Fasten eine solche von $110 \mu \mathrm{g}$ Aceton/l Alveolarluft (4). TAssopoulos und Mitarbeiter erhielten bei fünf adipösen Personen, welche 10 bis 36 Tage fasteten, Werte bis $200 \mu \mathrm{g}$ Aceton/l Alveolarluft (6). Aus der Arbeit von TAssopoulos geht jedoch nicht hervor, zu welchem Zeitpunkt innerhalb der Fastenperioden die genannten Werte bestimmt wurden. Die von uns bei Adipösen während vollständigem Fasten gefundenen Konzentrationen (Abb. 2) liegen im Bereich der von Rooth und von TASSOPOULOS mitgeteilten Resultate.

\section{Literatur}

1. Greenberg, L. A. und D. Lester, J. biol. Chemistry 154, 177 (1944). - 2. Glaubitt, D. und J. G. Rausch-Stroomann, Clin. chim. Acta (Amsterdam) 4, 165 (1959). - 3. StewART, R. D. und E. A. Boettner, N. England J. Med. 270, 1035 (1964). -
4. Rooth, G. und S. Ostenson, Lancet, London II/1966, 1102. 5. Klern, H. und J. Korzis, Medizinische, 345 (1958). - 6. TASsopoulos, C. N., D. Barnett und T. R. Fraser, Lancet, London $\mathrm{I} / 1969,1282$
Dr. H. Göschke Biirgerspital $\mathrm{CH} 4000$ Base] 